\title{
Editorial for the special issue: extreme events and vulnerability in environment and society
}

\author{
Thomas Glade • Carsten Felgentreff · Joern Birkmann
}

Received: 25 October 2010/Accepted: 25 October 2010/Published online: 10 November 2010

(C) Springer Science+Business Media B.V. 2010

Extreme events in nature and society are getting more and more important in our society. Hereby, it is not only important to address the "extremes" in terms of very large magnitude or intensity of a given natural process such as flood, heat wave, earthquake or landslide-it is also most important to consider the "extremes" in terms of societal impacts and societal consequences of natural hazards and creeping changes-including the degree of societal preparedness and response capacity to those impacts. Thus, it is indeed a major challenge to address the extremes in their completeness-and to summarize the different perspectives and approaches, but also to determine the major challenges when dealing with extreme events and its different facets.

This general topic has been addressed by three major events in 2007: a session within the biannual German Geographers Conferences, the meeting of the German Working Group of Natural Hazards and Risks and the international Expert Working Group on Measuring Vulnerability to Natural Hazards.

The Geography Conference organized under the umbrella of the German Association of Geography (DGfG) was carried out in Bayreuth, Germany with the arching conference topic "Handling Risks-disasters, destabilization, security". In this conference, Carsten Felgentreff and Thomas Glade were invited to organize a session on "Natural disasters and disaster prevention". Numerous invited experts in the field of natural disasters from natural and social science perspectives presented their view and experience on this topic. The session aimed to link vulnerability assessments with disaster prevention, thus to link social perception, communication and decision processes to hazard and disaster research based on natural science approaches. Hereby, it was envisaged to demonstrate the potential for disaster prevention. Specific addressed questions included: How can the change of

\author{
T. Glade $(\bowtie)$ \\ University of Vienna, Vienna, Austria \\ e-mail: thomas.glade@univie.ac.at \\ C. Felgentreff \\ University of Osnabrück, Osnabrück, Germany \\ J. Birkmann \\ UNU - EHS, Bonn, Germany
}


vulnerability be measured? Which levels are suitable for measurements? Who is responsible for reducing vulnerability, the individual or governmental agencies? How can spatial information be used in this discussion? Is it possible to move into new operational directions for dealing with vulnerability? How important are in this context debates on climate and environmental changes? All these questions were addressed by various presentations and followed up in the adjacent discussions.

The Working Group Natural Hazards and Risks, also part of the DGfG, organized in the same year a meeting on "Extreme Events in Environment and Society". This meeting was driven by the most recent disasters revealed by natural hazards - the Tsunami in the Indian Ocean in December 2004, the earthquake in Pakistan February 2005, Hurricane Katarina in the USA August 2005 and the rockslide in the Philippines in February 2006. Until today, the list could be extended. All these events demonstrate that the society has to tackle a new situation. Neither the events themselves nor the consequences are exactly predictable in the experienced circumstances. Instead, the processes are operating with previously unknown magnitude and intensity, but also the social relations show global affects of local events. The arising questions for this meeting were Are these extremes so devastating because they occur so infrequent? Or is the society not prepared, because they do not want to think the unthinkable? By the way, what is unthinkable? And for whom? These were some topics broadly discussed within the session of the Working Group.

The third occasion was a meeting of the international Expert Working Group on Measuring Vulnerability to Natural Hazards that is annually conducted and organized by the United Nations University-Institute for Environment and Human Security and chaired by PD Dr. Jörn Birkmann. The Expert Working Group Meeting in Bonn clearly stressed that we might have to revisit our dominant view on disasters. While disasters are to be avoided due to the human suffering, it was interesting to note that many changes in vulnerability and risk patterns were observed particularly following mega-disasters or extreme events in terms of societal impacts. Thus, a scientific discussion centered on the question whether disasters can also function as a catalyst of change and perhaps even allow for risk and vulnerability reduction. Particular attention was drawn to the post-disaster processes and their influence on vulnerability in the context of the Indian Ocean Tsunami especially in Sri Lanka and Indonesia and the Hurricane Katrina case in the USA. Most experts underlined that there is a necessity to understand disasters better not only in terms of impact and impact projection but also in terms of their positive and negative changes in organizations, institutions and policy; these are important aspects that future interdisciplinary research has to address. Furthermore, the Expert Working Group on Measuring Vulnerability focused on the question how tipping points of social-ecological systems in the context of disasters and vulnerability could be characterized and assessed. In many cases, extreme events are characterized by the destabilization of social and environmental conditions. Based on the presence of experts from the disaster risk reduction community (DRR) and experts from the ecological resilience community, it became clear that the discourses regarding disaster risk reduction and (socio-ecological) resilience need to be brought together and synergies need to be developed. This is a particular relevant for the broader understanding of risk, vulnerability and resilience. The topic of whether resilience is a new and useful concept was also heavily discussed particularly in terms of other concepts of development and the discourse about development strategies for developing countries.

Overall, the contributions from the three different venues fit quite well and examine from different perspective the different facets of extreme events and vulnerability. Therefore, it was agreed to publish a joined special issue on "Extreme events and 
vulnerability in Environment and Society" within the journal Natural Hazards. After more than 2 years of hard work, we are now able to present the selected contributions in this journal. The contributions are clustered in the three blocks natural events, vulnerability and social responses as well as integrated assessments. We purposely decided not to structure the manuscripts according to the three events, or even alphabetically according to the first authors surname-we rather preferred to demonstrate the overall and bridging themes by dividing the manuscripts based on the scientific content. In the following, the different papers are briefly summarized.

In the cluster natural events, Jörg Grunert and Sigrid Hess address for the Upper Middle Rhine Valley, one of the World Heritage sites, the problems associated with hazards and risks - in particular for floods and landslides. This valley has experienced numerous floods in the past, thus the population and stakeholders are aware of associated problems. In contrast, landslides are not so much in the public consciousness, although they have caused considerable damage to the infrastructure, and here mainly by the interruption of the main railway lines on both sides of the river. But landslide events are not limited to recent failures only. For example, the Kaub landslide of March 10, 1876 killed 28 persons. Such live threatening events have not occurred in the last decades; however, the lifelines are endangered. Since 2002, the German Rail (Deutsche Bahn, DB) stabilized all problematic slopes by steel ring nets. But despite these engineering structures, Grunert and Hess state that risk maps are still necessary for the Rhine valley in order to identify the most landslide-prone sites.

Roland Ngom and Alexander Siegmund present their research results on malaria risks. The authors explain that most of the existing spatial models use very broad spatial scalesmostly of continental or national scale. Commonly, these models do not cover the complexity of socioeconomic variables intervening into the malaria transmission process as well as malaria prevention strategies, which is most important when working in highly urbanized environments. This paper explores these shortcomings of the risk assessment of urban malaria. A set of environmental variables is suggested. This set was derived from remotely sensed and ground climate stations. The final ecological model is additionally based on GPS measurements and retrospective interviews. Various geoepidemiological and geosocial variables have been applied within multinomial logistic models to predict and evaluate the contribution of various variables to malaria risk. The authors were able to evaluate the contribution of ecologic, socio-ecologic, socioeconomic and behavioural characteristics for variables determining malaria risks.

In the first presentation in the cluster vulnerability and social responses, Koko Warner, Mohamed Hamza, Anthony Oliver-Smith, Fabrice Renaud and Alex Julca address deficits in our knowledge of the interplay of environmental change, ecological systems, socioeconomic vulnerability and impacts on spatial population movements, be it induced migration or population displacement. Migration can increase vulnerability, while in other cases, it might be an appropriate adaptation effectively minimizing vulnerability. Instead of simplistic causal explanations of environmentally induced migration, the authors argue in favour of the analysis of social, political, economic and environmental processes. Eventually, their paper mirrors the ambivalence of the current debate about environmental refugees/migrants: On the one hand, it is taken for granted that the world already has millions of people rightfully labelled as such, while on the other hand, it is admitted that it is somewhat difficult to define exactly what environmentally induced mobility means.

Martin Voss and Klaus Wagner focus on how stakeholders and societies can learn and do learn from small-scale disasters and emergencies. The author view disasters as a process that implies changes in social routines. Voss and Wagner outline that these learning 
processes rarely take place using three examples. They argue that there is a need to promote and strengthen self-organized and scale-independent learning processes following small disasters or emergencies linked to natural hazards. These learning processes should particularly be characterized by participative processes that involve people and stakeholders from various levels. In this context, the different causes that triggered a small disaster as well as with the intervention and mitigation measures that can be taken should be identified and evaluated. The authors conclude that more comprehensive and combined assessment strategies and tools would help policy makers to better adapt society to actual and future environmental change and potential disaster risks.

Christian Kuhlicke tackles the interrelation of the unknown and people's vulnerability. Inspired by a concept of ignorance meant as how people deal with their knowledge's limit, he suggests that studies of (social) vulnerability should take ignorance into account. Hence, social vulnerability is closely related to the discovery of one's own nescience, e.g. the unknown which - up to the moment of its discovery - has never been part of any conscious reflection. Accordingly, resilience is then one's ability to cope with the discovery of nescience. At times, the discovery of previous nescience happens (too) late: the situation becomes overwhelmingly, clearly indicating vulnerability of the people affected.

Terry Cannon and Detlef Müller-Mahn have contributed a conceptual paper discussing crucial aspects of adaptation and development within a changing climate context. They criticize the recent shift from vulnerability reduction to resilience thinking in academic and public debates. The concept of social vulnerability has a strong tradition in the realm of disaster risk reduction where it is widely accepted that social issues and processes (like power relations) determine one's exposure to risks. Stemming from ecosystem management, the idea of resilience might ostensibly depoliticise and foster technocratic interventions without any understanding of power relations and the behaviour of different actors with different levels of power. There are manifold contradictions between adaptation to climate change and development, and the authors do not just refer to constellations where economic growth makes people even more vulnerable. Hence, the authors argue in favour of adaptation to climate change in line with the reduction in vulnerability and poverty.

The contribution provided by Alex Julca and Oliver Paddison addresses some of the challenges that Small Island Developing States face in the context of climate change: development, vulnerability (with special reference to environment and economy) and outmigration. Well-known problems like little economic diversity, remoteness coupled with lack of infrastructure, dependency on remittances, scarcity of fresh and clean water and the like appear especially frightening to the authors before the background of climate change.

Disasters and impacts of extreme events cause major harm and losses, thus societies have to improve their measures to reduce disaster risk. Jörn Birkmann, Philip Buckle, Jill Jaeger, Mark Pelling, Neysa Setiadi, Matthias Garschagen, Nishara Fernando and Jürgen Kropp argue that disasters might also provide an opportunity for change and improvement if respective risk reduction measures are considered in the aftermath of the disaster. While much attention is given to the direct disaster impacts as well as relief and recovery operations, there has been little research on the characteristics and progress of change induced by disasters. According to the authors, change, as distinct from impacts, encompasses formal and informal responses to disasters events and their direct and indirect impacts. The paper develops an analytical framework for distinguishing change from disaster impacts. Based on research in Sri Lanka and Indonesia after the major Indian Ocean Tsunami disaster 2004, formal and informal changes are examined and discussed against the background of the conceptual framework. Different characteristics of 
organizational, institutional and political changes are discussed. The authors conclude that although organizational changes are often conducted after mega-disasters, fundamental interactions between society and nature are not sufficiently addressed. Hence, the authors argue that change-making processes after disasters need to be understood more in-depth in order to derive important strategic policy and methodological lessons learned for the future.

In the cluster integrated assessments, the first paper by Alexander Fekete, Marion Damm and Jörn Birkmann addresses scale questions, particularly aspects regarding spatial and temporal scales. It claims that these are often not sufficiently addressed within vulnerability and risk assessments. Hence, the authors argue that scale issues are a key aspect to better understand, structure and conduct vulnerability and risk assessments. Based on two practical case studies of vulnerability assessment in the context of river floods in Germany, the authors illustrate scale challenges linked to the development and conduction of vulnerability assessments at local and county level. The case studies illustrate that assessments on coarse scales often have to be based on existing statistical data for the respective units of analysis. In contrast, local assessments allow integrating new data particularly through household surveys. Among other benefits, local assessments can strengthen the knowledge of societal response capacities. Additionally, the authors show options to link assessments at local and county level and respective data from both a) household survey and b) statistical data. In this regard, selected key characteristics of vulnerability can be up-scaled, particularly aspects of susceptibility and coping capacities. The paper stresses the fact that more attention needs to be given to scale-specific patterns and characteristics of vulnerability in future research. Additionally, the scientific discussion about scales needs to address a broader spectrum of aspects than just technical issues - thus beside spatial scales, different temporal and functional scales need to be taken into consideration. Overall, the paper provides a systematic introduction into better understand and consider scale issues in risk and vulnerability assessment research linked to natural hazards, particularly floods.

Extreme weather events such as extreme floods or cyclones are not solely impacting societies, but also transforming social-ecological system that might move from one regime to another. The notion of what social-ecological systems (SES) are, and how this concept can help to better understand vulnerability and risk in the context of multiple thresholds and different triggers of regime shifts, is discussed in the paper by Fabrice Renaud, Jörn Birkmann, Marion Damm and Gilberto Gallopín. The authors argue that extreme events can cause a disruption of ecosystems and the services they provide to society, particularly if certain thresholds are reached. However, these thresholds are very difficult to evaluate because of the complex nature of SES. Based on an analysis conducted in the aftermath of the 2004 Indian Ocean tsunami impact on groundwater resources and the coastal communities in Sri Lanka, the authors illustrate the characterization of thresholds that could be identified in terms of the dependency of social groups with respect to essential ecosystem services. The authors develop a conceptual framework to better understand the behaviour of coupled social-ecological systems when subject to extreme events and external shocks, as well as when subject to potential changes or impacts, and when subject to subsystem components crossing a threshold. Therewith, the paper underlines that in future-in the context of climate change-SES might not solely move over a certain threshold when exposed to actual external shocks, but rather even the anticipation of certain impacts of climate change might trigger the social subsystem of an SES to cross a tipping point to a new state and to cause a regime shift of the SES thereafter. Overall, the authors underline the importance of improving the knowledge based regarding thresholds and shifts of SES 
from one domain to another in order to better understand risk and vulnerability in the context of extreme events.

All these different papers contribute to the overall topic of this Special Issue on "Extreme events and vulnerability in environment and society". We would like to thank numerous colleagues and friends, who contribute significantly to this special issue. First of all, our sincere thanks are for the management and editorial board of the journal. In particular, Ayrene Dialogue and Petra van Steenbergen, both from the editorial office of Springer, and Prof. Vladimir Schenk as the Supervising Editor provided guidance throughout the whole process. Numerous reviewers supported this issue through their thoughtful comments and suggestions and their very constructive criticism of the individual papers. We are fully aware that such reviews are always a voluntary service to the scientific community-besides all the demands in the own work place and the heavy workload. But we have experienced again that this service is of great value. The overall quality of the publications has been significantly increased. Finally, we would like to thank all the authors. We are aware that the publication process took a lot of time. However, the final result attests that it was worthwhile to wait. 